\title{
Lipid peroxidation product 4-hydroxynonenal as factor of oxidative homeostasis supporting bone regeneration with bioactive glasses ${ }^{\star}$
}

\author{
Lidija Mrakovcic', Renate Wildburger², Morana Jaganjac', Marina Cindric³, Ana Cipak1, \\ Suzana Borovic Sunjic' ${ }^{1}$, Georg Waeg ${ }^{4}$, Andrea M. Milankovic ${ }^{5}$ and Neven Zarkovic ${ }^{1 \times}$ \\ 'Rudjer Boskovic Institute, Department of Molecular Medicine, Zagreb, Croatia; ${ }^{2}$ Medical University, LKH Clinic of Traumatology, Graz, Austria; \\ 3Department of Pathology, Medical Faculty, Clinical Hospital Centre Zagreb, Croatia; ${ }^{4}$ Karl Franzens University, Institute of Molecular Biosciences, \\ Graz, Austria; ${ }^{5}$ Rudjer Boskovic Institute, NMR Center, Zagreb, Croatia
}

\begin{abstract}
Bone regeneration is a process of vital importance since fractures of long bones and large joints have a highly deleterious impact on both, individuals and society. Numerous attempts have been undertaken to alleviate this severe medical and social problem by development of novel bioactive materials, among which bioactive glass is the most attractive because of its osteoconductive and osteostimulative properties. Since lipid peroxidation is an important component of systematic stress response in patients with traumatic brain injuries and bone fractures, studies have been undertaken of the molecular mechanisms of the involvement of 4-hydroxynonenal (HNE), an end product of lipid peroxidation, in cellular growth regulation. We found that HNE generated in bone cells grown in vitro on the surfaces of bioactive glasses $45 \mathrm{S5}$ and $13-93$. This raises an interesting possibility of combined action of HNE and ionic bioglass dissolution products in enhanced osteogenesis probably through a mitogen-activated protein kinase (MAPK) pathway. While the proposed mechanism still has to be elucidated, the finding of HNE generation on bioglass offers a new interpretation of the osteoinducting mechanisms of bioglass and suggests the possibility of tissue engineering based on manipulations of oxidative homeostasis.
\end{abstract}

Keywords: bone regeneration, bioactive glass, lipid peroxidation, 4-hydroxynonenal, oxidative homeostasis

Received: 10 November, 2009; revised: 22 April, 2010; accepted: 14 May, 2010; available on-line: 31 May, 2010

\section{INTRODUCTION}

Bone is a dynamic, highly vascularized tissue with a unique internal repair capacity to heal and remodel without scarring (Buckwalter et al., 1996a; 1996b). Skeletal development and fracture repair includes the coordination of multiple events such as migration, differentiation, and activation of multiple cell types and tissues (Colnot et al., 2005).

The molecular events that govern fracture healing are a complex network of signals signifying tissue damage, cell death, cell recruitment, cell proliferation, cell differentiation, and tissue formation (Dimitriou et al., 2005). The phases of fracture healing can be summarized as: hematoma formation, inflammation, angiogenesis, car- tilage/callus formation, and bone remodeling. Different sets of molecules interact with both local cells and circulating cells to coordinate the healing cascade: effectors of inflammation (IL-1, IL-6, COX-2), mitogens (transforming growth factor beta (TGF $\beta$ ), insulin-like growth factor (IGF), fibroblast growth factor (FGF) and platelet derived growth factor (PDGF)), morphogens (bone morphogenetic proteins (BMPs)), and angiogenic factors (vascular endothelial growth factor (VEGF) and angiopoietins).

Bone fractures, especially of long bones or large joints, are a major medical disorder and are often not followed by successful recovery particularly in elderly patients, in patients that suffer from multifragmentary bone fractures or in polytraumatized patients. In a large number of cases the resulting skeletal deficiencies require surgical intervention and repair. Currently three different graft types are used for reconstructive surgery: autogenic grafts, allogenic grafts, and bone substitutes. Because of some drawbacks of autografts and allografts, like donor site morbidity, limited donor bone supply, rejection phenomena and risk of viral infection, a wide range of bioactive materials as calcium phosphate ceramics (Ca-P), bioactive glasses (BG) and bioactive glass-ceramics have been introduced. A bioactive material is by definition "one that elicits a specific biological response at the interface of the material that results in the formation of a bond between the tissues and the material" (Hench et al., 1971). These materials generate a carbonated hydroxyapatite layer that is equivalent chemically and structurally to the biological mineral of the bone, which is known to be the determining step for biointegration (Kitsugi et al., 1987). Furthermore, it has been found that bioactive glasses containing less than $55 \% \mathrm{SiO}_{2}$ exhibit not only osteoconductivity (able to support abundant bone formation), but are also responsible for osteoproduction by stimulating proliferation and differentiation of osteo-

\footnotetext{
e-mail: zarkovic@irb.hr

* Presented at the COST B-35 Work Group 4 Open Workshop "Natural and synthetic antioxidants", September 25-26, 2009, Rzeszów Poland.

Abbreviations: ERK, extracellular signal regulated kinase; FGF fibroblast growth factor; HNE, 4-hydroxynonenal; IGF, insulin-like growth factor; JNK, Jun $\mathrm{NH}_{2}$-terminal kinase; MAPK, mitogenactivated protein kinase; NF- $K B$, nuclear factor- $K B$; PDGF, platelet derived growth factor; PKC, protein kinase $C$; ROS, reactive oxygen species; TGF $\beta$, transforming growth factor beta; VEGF, vascular endothelial growth factor.
} 
progenitor cells (Ohgushi et al., 1996). A major bonebioactive material is the 45S5 bioactive glass (Hench et al., 1971). It is highly bioactive and both osteoinductive (able to induce bone formation) and osteoconductive. Moreover, it has been shown that the ionic dissolution products of $45 \mathrm{~S} 5$ Bioglass may enhance new bone formation (osteogenesis) through direct control of genes that regulate cell induction and proliferation (Xynos et al., 2000). A recent report demonstrates that the dissolution products of $45 \mathrm{~S} 5$ Bioglass create an extracellular environment that is capable of supporting osteoblast phenotype expression and extracellular matrix deposition and mineralization in vitro in human fetal osteoblasts (Tsigkou et al., 2009). There are also indications from previous reports of in vivo studies (Day et al., 2004) that bioactive glass (45S5 Bioglass) enhances vascularization of tissue engineering constructs.

Another silicate-based bioactive glass that we examined in our research is the 13-93 bioactive glass with a composition slightly modified relative to that of $45 \mathrm{~S} 5$. The 13-93 glass supports in vitro growth and differentiation of MC3T3-E1 preosteoblastic cells (Fu et al., 2008), and quantitative measurement of DNA showed no significant differences in cell proliferation induced by dense disks of 45S5 and 13-93 glass (Brown et al., 2008).

On the other hand, our previous research has revealed a connection between stress response and the phenomenon of enhanced osteogenesis in patients with traumatic brain injury. These patients are characterized by a very short period of bone healing followed by heterotopic ossification or hypertrophic callus formation. This stress response involves hormonal imbalance associated with changing growth factor activities, in particular bFGF (Wildburger et al., 1994; 1995) and IGF-1 (Wildburger et al., 2001), together with oxidative stress and lipid peroxidation (Wildburger et al., 1997; 2000). Thus, lipid peroxidation is an important parameter of systemic response in patients with traumatic brain injuries and with bone fractures.

Lipid peroxidation occurs when reactive oxygen species (ROS) generated during oxidative stress damage lipids mostly present in the plasma, mitochondrial, and endoplasmic reticulum membranes. One of the major toxic products generated during lipid peroxidation, considered as "second messenger of free radicals", is the $\alpha, \beta$-unsaturated aldehyde 4-hydroxynonenal (HNE), which is derived from $\omega-6$ polyunsaturated fatty acids such as arachidonic acid and linoleic acid (Esterbauer et al., 1991). Recent results have shown that HNE acts as a growth-regulating factor interfering with the activity of cytokines and may affect the growth of cultured human bone cells (Sunjic Borovic et al., 2005; Borovic et al., 2007a). HNE is a major bioactive marker of lipid peroxidation (Zarkovic, 2003) that acts as a second messenger of free radicals and a signaling molecule interfering with the activities of various signal kinases, such as protein kinase $\mathrm{C}(\mathrm{PKC})$ and mitogen-activated protein kinases (MAPKs), to regulate cellular processes from proliferation to differentiation and apoptosis (Leonarduzzi et al., 2004). Because of its involvements, HNE is considered

Table 1. Composition of bioactive glasses 4555 and 13-93

\begin{tabular}{lllllll}
\hline Typical compositions (wt \%) & $\mathrm{SiO}_{2}$ & $\mathrm{Na}_{2} \mathrm{O}$ & $\mathrm{K}_{2} \mathrm{O}$ & $\mathrm{CaO}$ & $\mathrm{P}_{2} \mathrm{O}_{5}$ & $\mathrm{MgO}$ \\
\hline $45 \mathrm{~S} 5$ & 45 & 24.5 & - & 24.5 & 6 & - \\
$13-93$ & 53 & 6 & 12 & 20 & 4 & 5 \\
\hline
\end{tabular}

wt, weight as a particularly interesting biomolecule that might play an important role in regulating regeneration of damaged tissue such as bone.

Since our previous research had shown that HNE has a role in differentiation, apoptosis and cell proliferation (Sunjic Borovic et al., 2005) in human osteoblast cells and can increase cell growth in physiological concentrations (Borovic et al., 2007b) we wanted to find out whether HNE-protein adducts are formed in human osteosarcoma (HOS) cells in contact with bioactive glasses during bone growth in vitro.

\section{MATERIALS AND METHODS}

Bioactive glass. Bioactive glasses $45 \mathrm{~S} 5$ and $13-93$ (supplied by Mo-Sci Corporation, USA, $10 \mathrm{~cm}$ long rods of $10 \mathrm{~mm}$ diameter) were cut into $1 \mathrm{~mm}$ thick discs (composition of both bioactive glasses is shown in Table 1). The discs were sterilized in $70 \%$ ethanol, left to dry in a laminar flow hood and treated under UV light for $20 \mathrm{~min}$. After sterilization the discs were put in a $24-$ well plate (TPP, Switzerland).

HOS cells. The human osteosarcoma cell line HOS was obtained from American Type Culture Collection (ATCC). Cells were maintained in DMEM (Dulbecco's modified Eagle's medium, Sigma, USA) with $10 \%$ (v/v) foetal calf serum (FCS, Sigma, USA) in an incubator (Heraeus, Germany) at $37^{\circ} \mathrm{C}$, with a humid air atmosphere containing $5 \% \mathrm{CO}_{2}$. The cells were detached from semiconfluent cultures with a $0.25 \%(\mathrm{w} / \mathrm{v})$ trypsin solution for $5 \mathrm{~min}$. Viable cells (upon Trypan Blue exclusion) were counted in a Bürker-Türk hemocytometer and used for experiments.

Immunocytochemistry. Cells were seeded at $2 \times 10^{4}$ cells in $100 \mu \mathrm{l}$ of medium on the upper side of bioglass specimens 45S5 and 13-93 (concentration: 2.55 $\times 10^{4}$ cells $/ \mathrm{cm}^{2}$ ) and left for $3 \mathrm{~h}$ to attach before adding media to cover the glass discs. Cell cultures developed on standard histological glass served as controls. Quadruplicates of cultures were prepared for immunostaining performed on the 3rd day of cell culturing.

Immunostaining for HNE-modified proteins was carried out on formalin-fixed cell samples with monoclonal antibodies obtained from culture medium of clone "HNE 1g4", produced by fusion of Sp2-Ag8 myeloma cells with B-cells of a BALB-c mouse immunized with HNE-modified keyhole limpet hemocyanine. The antibody is specific for the HNE-histidine epitope in HNEprotein (peptide) conjugates and gives only $5 \%$ cross reactivity with $\mathrm{HNE}$-lysine and $4 \%$ with $\mathrm{HNE}$-cysteine (Waeg et al., 1996).

Immunostaining was performed as described before (Zarkovic et al., 1997) using LSAB kit (DAKO, Denmark). Briefly, samples were incubated with anti-HNE monoclonal antibodies (dilution $1: 10$ ) for $2 \mathrm{~h}$ in humid chambers at room temperature, washed three times for 5 min with phosphate-buffered saline (PBS) and then incubated with biotinylated secondary antibody for $30 \mathrm{~min}$. After washing with Tris-buffered saline (TBS), three times for $5 \mathrm{~min}$, samples were incubated with streptavidin peroxidase for $30 \mathrm{~min}$. Finally, the reaction was visualized with DAB (3,3-diaminobenzidine tetrahydrochloride in organic solvent; DAKO, Denmark) giving a brown color that indicated the presence of HNE. Negative control was done without application of HNE-histidine specific mono- 
clonal antibodies. Qualitative evaluation of differences in DAB staining intensity was done by inverted microscopy (Krüss Optotronic, Germany). Although quadruplicates of cultures were prepared for the immunostaining, due to the tissue-like multilayer growth pattern of cells cultured on bioglass, instead of usual monolayer growth, quantitative analyses of the amounts of developed HNEprotein adducts could not be done.

Analyses of ROS production. After seeding at a density of $2 \times 10^{4}$ cells/bioglass disc, cells were incubated for $72 \mathrm{~h}$. Quadruplicates of cultures were prepared for the analysis performed on the 3rd day of cell culturing. On the third day of cell cultivation, a nonfluorescent probe for intracellular ROS detection, 2',7'-dichlorofluorescin diacetate (DCFH-DA, Fluka), was added to cell medium to a final concentration of $10 \mu \mathrm{M}$. This cell-permeable dye remains nonfluorescent inside the cell until the acetate groups are removed by intracellular esterases and oxidized by intracellular ROS to the fluorescent compound 2',7'-dichlorofluorescein (DCF) which can be detected as a measure of intracellular ROS. Fluorescence intensity was observed with a microscope (Zeiss Axiovert 25).

\section{RESULTS AND DISCUSSION}

Immunochemical analysis revealed that during incubation of human bone cells in vitro on bioactive glasses 45S5 and 13-93, HNE is generated in the cells, unlike in cells growing on normal histological glass (Fig. 1). This suggests a possible physiological role of HNE in bone regeneration process not associated with cytotoxicity because the cells were proliferating (increasing their number) during cultivation period (not shown) as previously reported (Xynos et al., 2000; Brown et al., 2008). Moreover, since HNE is considered a "second messenger of free radicals", oxidative stress and lipid peroxidation should not only be considered as generators of biomolecular damage, and thus important contributors to aging as well as causative factors of diseases, but also as possible mediators of normal physiological functions (Wildburger et al., 2009). That allowed consideration of oxidative stress also as a physiological process introducing new term "oxidative homeostasis", which implies involvement of reactive oxygen species (ROS) and lipid peroxidation in various physiological processes (Zarkovic et al., 2009). In favor of this possibility is also the atypical growth pattern observed when HOS cells were cultured on bioglass. Namely, the cells grown on standard histological glass formed a typical monolayer and did not produce endogenous HNE-protein adducts, while those grown on bioglass formed a tissue-like multilayer producing abundant HNE (Fig. 1). The disadvantage of thus enhanced tissue-like growth of the HOS cells cultured on bioglass unfortunately limited the possibility of quantitative analyses of the amount of HNE-protein adducts formed. The rigidity of the tissue-like structures formed by HOS cells grown on bioglass did not allow their removal as intact cells or cell lyses that would allow semiquantitative immunocytochemical analyses or quantitative analyses using enzyme-linked immunosorbent assay for the HNE-histidine adducts based on the use of the same monoclonal antibodies as was done before for the HOS cells cultured under standard conditions (Borovic et al., 2006). However, since no HNE-histidine adducts were detected in cells cultured on normal histological glass, but only if the cells were grown on bioglass, we could conclude that the growth of the HOS cells in a tissue-like pattern on bioglass was associated with production of $\mathrm{HNE}$, known to act as a growth-regulating factor and signaling molecule considered also as a second messenger of free radicals.

To further reveal if the HOS cells grown on bioglass undergo physiological oxidative stress manifested by ROS and HNE production, we determined endogenous ROS production during cell growth on the bioactive glass by a specific fluorescence method (DCF, Fig. 2). As can be seen in Fig. 2, ROS may trigger lipid peroxidation and generation of $\mathrm{HNE}$, which is in turn able to induce ROS production, which usually leads to the self-catalysed process of lipid peroxidation that could destroy the cells. However, in our study it appeared to enhance the growth of HOS cells on bioglass. Therefore, we would like to offer some possible explanations why HNE-protein adducts could be found in bone cells grown on the bioglass surface in vitro, keeping in mind that further studies are necessary to fully explain the observed beneficial effects of the endogenous oxidative stress and lipid peroxidation occurring in HOS cells growth on bioglass.

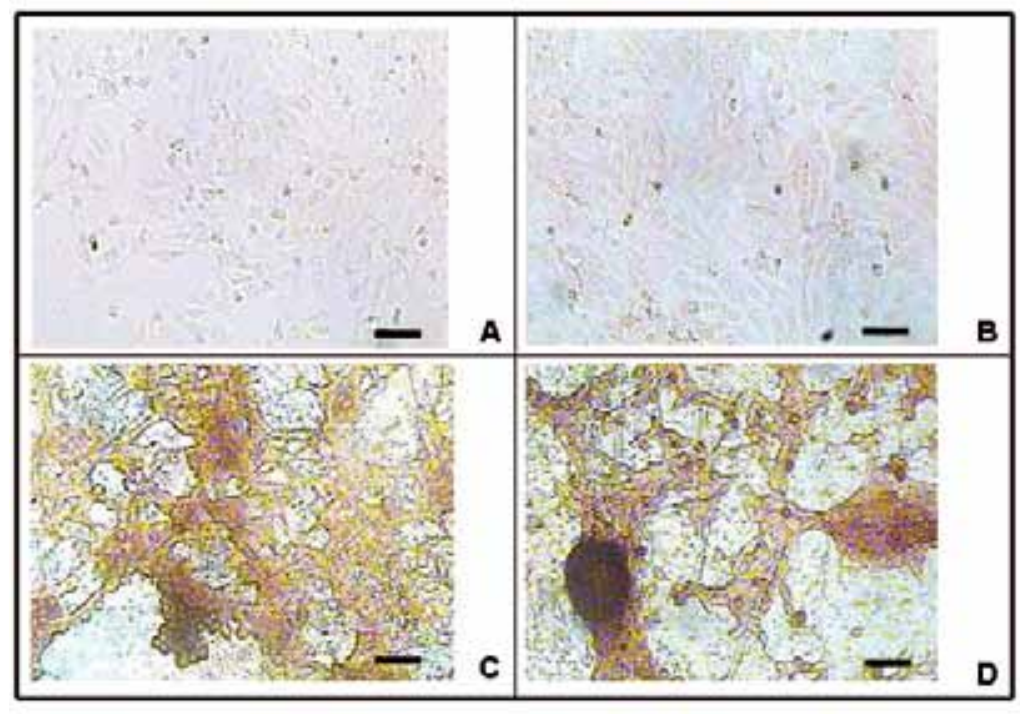

Figure 1. Immunochemical detection of HNEhistidine adducts.

The aducts were detected using specific monoclonal antibodies after three days of cultivation of HOS cells on standard histological glass or on bioglass (scale bar $50 \mu \mathrm{m}$ ), as detailed in Materials and Methods. (A) HOS cells cultured on standard histology glass - negative control (without primary antibody); (B) HOS cells cultured on normal standard histology glass; (C) HOS cells cultured on bioactive glass 45S5; (D) HOS cells cultured on bioactive glass 13-93. Cells grown on standard histological glass formed typical monolayer cultures and did not produce endogenous HNE-protein adducts, while those grown on bioglass formed a tissue-like multilayer producing abundant HNE. Representative images of quadruplicate cultures are presented. 


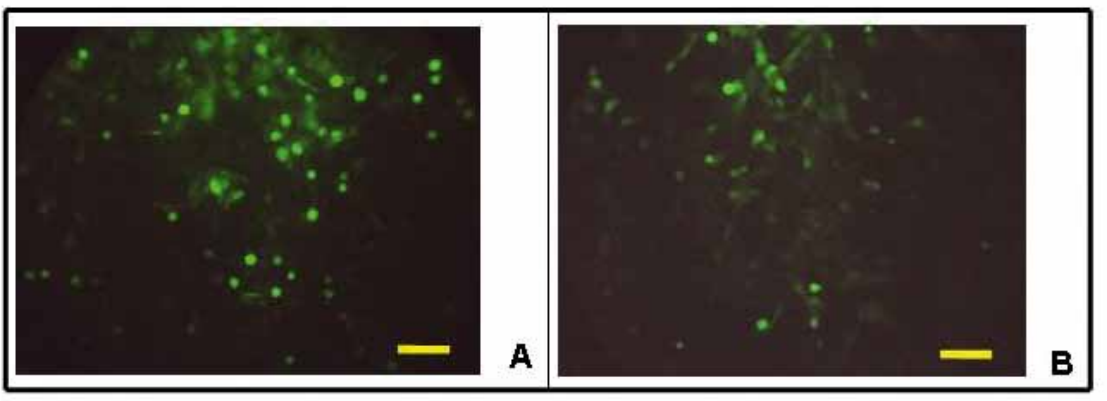

Figure 2. Detection of intracellular reactive oxygen species produced by HOS cells cultured on bioactive glass for 3 days.

Reactive oxygen species (ROS) were visualised by fluorescence using 2',7'dichlorofluorescein (DCF) (scale bar $100 \mu \mathrm{m})$. HOS cells cultured on bioactive glass 45S5 HOS cells cultured on bioactive glass 13-93. Representative images of quadruplicate cultures are presented.
There is growing evidence for physiological generation of ROS that can occur as a byproduct of other biological reactions. NADPH oxidase is known to be the major source of ROS, but ROS generation also occurs with mitochondria, peroxisomes, cytochrome P450, and other cellular components (Bedard \& Krause, 2007). Several growth factors and cytokines binding to different types of cell membrane receptors can elicit a rise in intracellular ROS. It has been found that generation of ROS is induced with TGF $\beta$ in mouse osteoblastic cells and in human lung fibroblasts (Kamata \& Hirata, 1999) and with PDGF in human lens epithelial cells (Chen et al., 2007). Furthermore, it has been reported that ROS activate MAPK pathways in several systems, including extracellular signal regulated kinase (ERK)1/2, Jun $\mathrm{NH}_{2}-$ terminal kinase and P38 MAPK cascades (Henrotin et al., 2003).

On the other hand, intracellulary generated ROS induce lipid peroxidation and formation of reactive aldehydes of which HNE is one of significance. When present in the cell, HNE forms stable covalent HNE-protein adducts by reaction with cysteine, lysine and histidine residues (Zarkovic, 2003; Uchida, 2003). These protein adducts were found in normal skeletal development during embryogenesis (Yan \& Hales, 2006) and in vari- ous tissues of animal and human origin (Zarkovic et al., 2009). The impact of HNE on the fate of the cell is dose-dependent; at low levels, HNE promotes cell proliferation, whereas at higher concentrations it induces cell cycle arrest, differentiation, and finally apoptosis (Awasthi et al., 2005). Exposure to HNE induces vascular smooth muscle growth, accompanied by the activation of MAPKs (ERK, c-Jun $\mathrm{NH}_{2}$-terminal kinase, and p38), the induction of c-fos and c-jun gene expression, and activator protein 1 (AP-1) DNA binding activity (Kakishita \& Hattori, 2001). Others have reported that HNE conjugates of glutathione, the most important cellular antioxidant involved in HNE detoxification, activate PKC and stimulate nuclear factor- $x \mathrm{~B}(\mathrm{NF}-x \mathrm{~B})$ and $\mathrm{AP}-1$-dependent gene transcription leading to cell proliferation (Ramana et al., 2006). Furthermore, recent observations show that stimulation with $\mathrm{HNE}$ at sublethal concentrations induces adaptive response and enhances cell tolerance, thereby protecting cells against the forthcoming oxidative stress (Niki, 2009).

On the other hand, bioglass ionic dissolution products were found to stimulate several growth factors including IGF-II, a key regulator of osteoblast homeostasis, and VEGF, a member of the fibroblast growth factor family with osteogenic potential. Several intracellular signal-

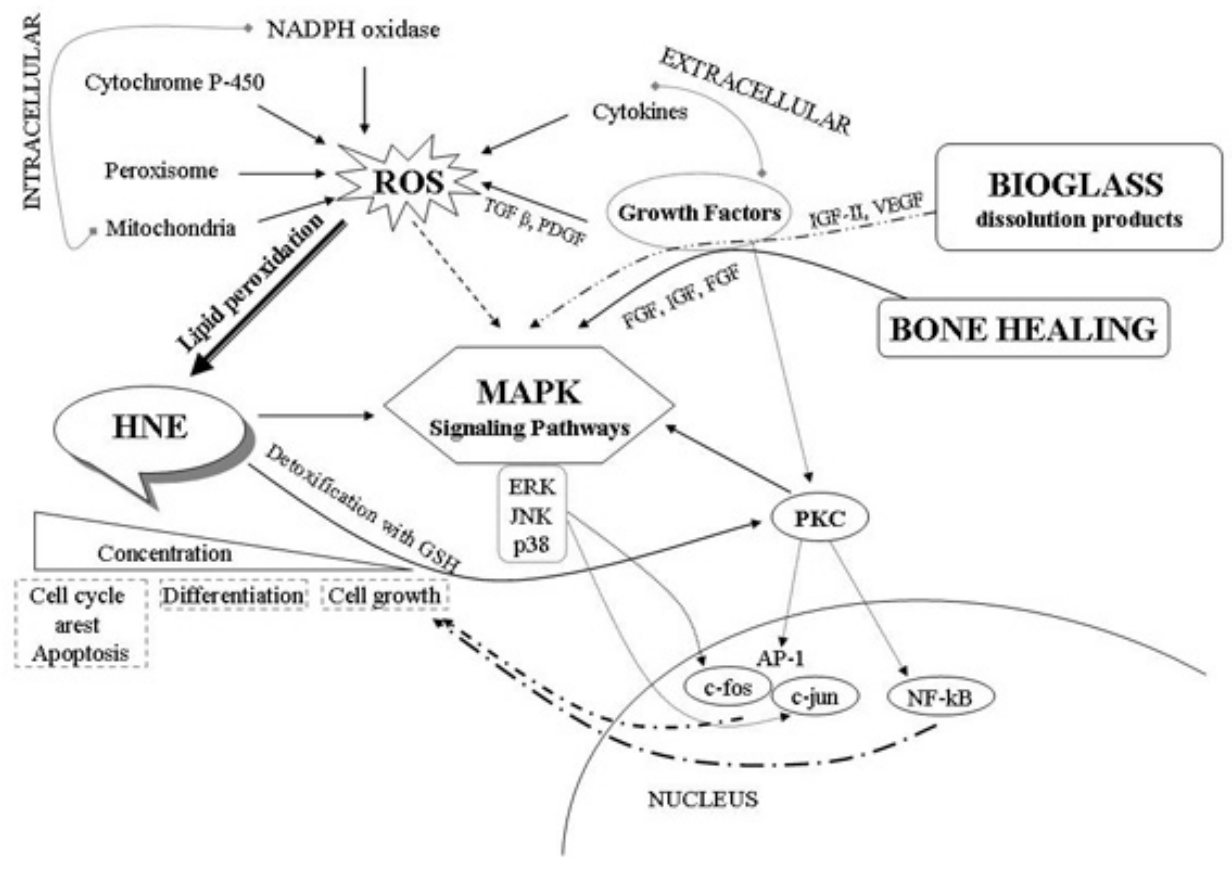

Figure 3. Proposed combined effect of bioglass dissolution products, ROS and HNE on cell growth and thus bone regeneration, mediated through MAPK signaling pathways. 
ing molecules are involved in osteoblast activation due to IGF-II and VEGF, including the ERK and MAPK pathways. Four kinases have been shown to be induced by the treatment, MAPKAP kinase-2, MAPK-2, MAPK p38, and ERK1 (Xynos et al., 2000).

We should also mention the complexity of the biological response involved in bone regeneration. Several mechanisms have been reported, like the Indian hedgehog (Ihh) pathway, bone morphogenetic protein (BMP) pathway, canonical Wnt- $\beta$-catenin pathway and MAPK pathway the most interesting from our point of view. The MAPK pathway was found to be an obligatory transducer crucial for post-fracture bone healing because it transduces signals from several growth factors (FGF, IGF, EGF) (Deschaseaux et al., 2009).

So, taking all the above into consideration, we propose a combined effect of bioglass dissolution products and $\mathrm{HNE}$ in bone regeneration during fracture healing and suggest an involvement of the MAPK pathway (Fig. 3).

Consequently, we speculate that bioglass functionalized with physiological concentrations of $\mathrm{HNE}$ prior to its implantation in damaged bone tissue could more efficiently trigger signaling reactions and enhanced osteogenesis. Although more investigation should be done, a report of a successful use of carbon nanotubes functionally activated with $\mathrm{HNE}$ for stimulation of neuronal growth in vitro (Mattson et al., 2000) is encouraging.

\section{Acknowledgement}

This research was supported by the Croatian Ministry of Science, Education and Sport, by the Austrian National Bank Jubilaeums Fund and by COST Action B35.

\section{REFERENCES}

Awasthi YC, Ansari GA, Awasthi S (2005) Regulation of 4-hydroxynonenal mediated signaling by glutathione $S$-transferases. Methods Ensymol 401: 379-407.

Bedard K, Krause KH (2007) The NOX family of ROS-generating NADPH oxidases: physiology and pathophysiology. Physiol Rev 87: 245-313.

Borovic S, Rabuzin F, Waeg G, Zarkovic N (2006) Enzyme-linked immunosorbent assay for 4-hydroxynonenal-histidine conjugates. Free Radic Res 40: 809-820.

Borovic S, Cipak A, Meinitzer A, Kejla Z, Perovic D, Waeg G, Zarkovic N (2007a) Differential sensitivity to 4-hydroxynonenal for normal and malignant mesenchymal cells. Redox Rep 12: 50-54.

Borovic S, Sunjic I, Wildburger R, Mittelbach M, Waeg G, Zarkovic $N$ (2007b) Lipid peroxidation end product as a modulator of cell growth. In Progress in cell growth process research. Hayashi $\mathrm{T}$, ed, pp 187-208. Nova Science Publishers Inc, New York.

Brown RF, Day DE, Day TE, Jung S, Rahaman MN, Fu Q (2008) Growth and differentiation of osteoblastic cells on 13-93 bioactive glass fibers and scaffolds. Acta Biomater 4: 387-396.

Buckwalter JA, Glimcher MJ, Cooper RR, Recker R (1996a) Bone biology. I: Structure, blood supply, cells, matrix, and mineralization. Instr Course Lect 45: 371-386.

Buckwalter JA, Glimcher MJ, Cooper RR, Recker R (1996b) Bone biology. II: Formation, form, modeling, remodeling, and regulation of cell function. Instr Course Lect 45: 387-399.

Chen ZH, Saito Y, Yoshida Y, Sekine A, Noguchi N, Niki E (2005) 4-Hydroxynonenal induces adaptive response and enhances PC12 cell tolerance primarily through induction of thioredoxin reductase 1 via activation of Nrf2. I Biol Chem 280: 41921-41927.

Chen KC, Zhou Y, Zhang W, Lou MF (2007) Control of PDGF-induced reactive oxygen species (ROS) generation and signal transduction in human lens epithelial cells. Mol Vis 13: 374-387.

Colnot C, Romero DM, Huang S, Helms JA (2005) Mechanisms of action of demineralized bone matrix in the repair of cortical bone defects. Clin Orthop Relat Res 435: 69-78.

Day RM, Boccaccini AR, Shurey S, Roether JA, Forbes A, Hench LL, Gabe SM (2004) Assessment of polyglycolic acid mesh and bioac- tive glass for soft-tissue engineering scaffolds. Biomaterials 25: 58575866.

Deschaseaux F, Sensébé L, Heymann D (2009) Mechanisms of bone repair and regeneration. Trends Mol Med 15: 417-429.

Dimitriou R, Eleftherios T, Giannoudis P (2005) Current concepts of molecular aspects of bone healing. Injury 36: 1392-1404.

Esterbauer H, Schaur RJ, Zollner H (1991) Chemistry and biochemistry of 4-hydroxynonenal, malonaldehyde and related aldehydes. Free Radic Biol Med 11: 81-128.

Fu Q, Rahaman MN, Bal BS, Brown RF, Day DE (2008) Mechanical and in vitro performance of 13-93 bioactive glass scaffolds prepared by a polymer foam replication technique. Acta Biomater 4: 1854-1864.

Hench LL, Splinter RJ, Greenlee TK, Allen WC (1971) Bonding mechanisms at the interface of ceramic prosthetic materials. I Biomed Mater Res Symp 2: 117-141.

Henrotin YE, Bruckner P, Pujol JP (2003) The role of reactive oxygen species in homeostasis and degradation of cartilage. Osteoarthritis Cartilage 11: 747-755.

Kakishita H, Hattori Y (2001) Vascular smooth muscle cell activation and growth by 4-hydroxynonenal. Life Sci 69: 689-697.

Kamata H, Hirata H (1999) Redox regulation of cellular signalling. Cell Signal 11: 1-14.

Kitsugi T, Nakamura T, Yamamura T, Kokubu T, Shibuya T, Takagi M (1987) SEM-EPMA observation of three types of apatite-containing glass-ceramics implanted in bone: the variance of a $\mathrm{Ca}-\mathrm{P}-$ rich layer. J Biomed Mater Res 21: 1255-1271.

Leonarduzzi G, Robbesyn F, Poli G (2004) Signaling kinases modulated by 4-hydroxynonenal. Free Radic Biol Med 37: 1694-1702.

Mattson MP, Haddon RC, Rao AM (2000) Molecular functionalization of carbon nanotubes and use as substrates for neuronal growth. $J$ Mol Neurosci 14: 175-182.

Niki E (2009) Lipid peroxidation: physiological levels and dual biological effects. Free Radic Biol Med 47: 469-484.

Ohgushi H, Dohi Y, Yoshikawa T, Tamai S, Tabata S, Okunaga K, Shibuya T (1996) Osteogenic differentiation of cultured marrow stromal stem cells on the surface of bioactive glass ceramics. $J \mathrm{Bi}$ omed Mater Res 32: 341-348.

Ramana KV, Bhatnagar A, Srivastava S, Yadav UC, Awasthi S, Awasthi YC, Srivastava SK (2006) Mitogenic Responses of Vascular Smooth Muscle Cells to Lipid Peroxidation-derived Aldehyde 4-Hydroxytrans-2-nonenal (HNE). J Biol Chem 281: 17652-17660.

Sunjic Borovic S, Cipak A, Rabuzin F, Wildburger R, Zarkovic N (2005) The influence of 4-hydroxy-2-nonenal on proliferation, differentiation and apoptosis of human osteosarcoma cells. Biofactors 24: $141-148$.

Tsigkou O, Jones JR, Polak JM, Stevens MM (2009) Differentiation of fetal osteoblasts and formation of mineralized bone nodules by 45S5 Bioglass conditioned medium in the absence of osteogenic supplements. Biomaterials 30: 3542-3550.

Uchida K (2003) 4-Hydroxy-2-nonenal: a product and mediator of oxidative stress. Prog Lipid Res 42: 318-343.

Waeg G, Dimisity G, Esterbauer H (1996) Monoclonal antibodies for detection of 4-hydroxynonenal modified proteins. Free Radic Res 25: 149-159.

Wildburger R, Zarkovic N, Egger G, Petek W, Zarkovic K, Hofer HP (1994) Basic fibroblast growth factor (bFGF) immunoreactivity as a possible link between head injury and impaired bone fracture healing. Bone Miner 27: 183-192.

Wildburger R, Zarkovic N, Egger G, Petek W, Meinitzer A, Borovic S, Zarkovic K, Li L, Stipancic I, Trbojevic-Cepe M, Cvoriscec D, Doko M (1995) Comparison of the values of basic fibroblast growth factor determined by an immunoassay in the sera of patients with traumatic brain injury and enhanced osteogenesis and the effects of the same sera on the fibroblast growth in vitro. Eur J Clin Chem Clin Biochem 33: 693-698.

Wildburger R, Zarkovic N, Tatzber F, Borovic S, Stipancić I, Kejla Z (1997) Post-traumatic dynamic changes of the titer of autoantibodies oxidized low-density lipoproteins; unspecific or organ-specific consequences of injury. Biofactors 6: 292-293.

Wildburger R, Borovic S, Zarkovic N, Tatzber F (2000) Post-traumatic dynamic changes of the antibody titer against oxidized low-density lipoproteins. Wien Klin Wochenschr 112: 798-803.

Wildburger R, Zarkovic N, Leb G, Borovic S, Zarkovic K, Tatzber F (2001) Post-traumatic changes of insulin like growth factor type 1 and growth hormone in patients with bone fractures and traumatic brain injury. Wien Klin Wochenschr 113: 119-126.

Wildburger R, Mrakovcic L, Stroser M, Andrisic L, Sunjic SB, Zarkovic K, Zarkovic N (2009) Lipid peroxidation and age-associated diseases-cause or consequence? Turk. Klin Tip Bilim Derg 29: 189-193.

Xynos ID, Edgar AJ, Buttery LD, Hench LL, Polak JM (2000) Ionic dissolution products of bioactive glass increase proliferation of human osteoblasts and induce insulin-like growth factor II mRNA expression and protein synthesis. Biochem Biophys Res Commun 276: $461-465$. 
Yan J, Hales BF (2006) Depletion of glutathione induces 4-hydroxynonenal protein adducts and hydroxyurea teratogenicity in the organogenesis stage mouse embryo. J Pharmacol Exp Ther 319: 613-621.

Zarkovic, N (2003) 4-Hydroxynonenal as a bioactive marker of pathopysiological processes. Mol Aspects Med 24: 281-291.

Zarkovic K, Zarkovic N, Schlag G, Redl H, Waeg G (1997) Histological aspects of sepsis induced brain changes in a baboon model. In: Shock, sepsis and organ failure. Schlag G, Redl H, Traber DL, eds, pp 146-160. 5th Wiggers Bernard Conference, Springer-Verlag, Heidelberg.
Zarkovic N, Cipak Gasparovic A, Cindric M, Waeg G, Borovic Sunjic S, Mrakovcic L, Jaganjac M, Kolenc D, Andrisic L, Gveric Ahmetasevic S, Katusic A, Cherkas A, Juric Sekhar G, Wildburger R, Zarkovic K (2009) 4-Hydroxynonenal-protein adducts as biomarkers of oxidative stress, lipid peroxidation and oxidative homeostasis. In Proceedings of the European Society for Free Radical Research Congress. Caporossi D, Pigozzi F, Sabatini S, eds, pp 37-45. Medimont Srl, Rome, Bologna. 